\title{
Determination of L-Ascorbic Acid by a Chemiluminescence Method using a Metal-MWCNT Immobilized Microfluidic Chip
}

\author{
Sang Hak Lee ${ }^{1}$, So Yeon Kim ${ }^{1}$, Hae Jin Jo ${ }^{1}$, Kyung Min Kim ${ }^{1}$, Gyu-Man Kim², Trung Dung Dang ${ }^{2}$, \\ Yeoun Suk Suh', Young Ho Kim ${ }^{3}$ \\ ${ }^{1}$ Department of Chemistry, Kyungpook National University, Daegu, 702-701, South Korea \\ ${ }^{2}$ School of Mechanical Engineering, Kyungpook National University, Daegu, 702-701, South Korea \\ ${ }^{3}$ Research Institute of Advanced Energy Technology, Kyungpook National University, Daegu, 702-701, \\ South Korea \\ E-mail:shlee@knu.ac.kr
}

\begin{abstract}
The determination of L-ascorbic acid (AA) by a high throughput chemiluminescence (CL) method using a metal ion attached multiwalled carbon nanotubes (MWCNTs) immobilized microfluidic chip has been developed. The method is based on enhancing CL intensity of the luminol-hydrogen peroxide system with the immobilized metal-MWCNTs in the microfluidic chip and on the quenching of $\mathrm{CL}$ intensity with addition of $\mathrm{AA}$. The effects of $\mathrm{pH}$, concentrations of luminol, hydrogen peroxide and metal ions on the $C L$ intensity were investigated and optimized. The calibration curve for AA was linear over the range of $1.6 \times 10^{-8} \mathrm{M}$ to $6.4 \times 10^{-7} \mathrm{M}$, the correction coefficient was 0.98913 and the detection limit was $1.11 \times 10^{-9} \mathrm{M}$.
\end{abstract}

Key words: Metal-MWCNTs, Microfluidic chip, L-ascorbic acid, chemiluminescence

\section{Introduction}

L-ascorbic acid (AA, (5R)-[(1S)-1,2dihydroxyethyl]-3,4-dihydroxyfuran-2(5H)-one) act as an antioxidant in various biochemical pathways [1]. Various determination methods including spectrophotometric and electrochemical methods were reported to determine AA in food or pharmaceutical samples [2,3]. Recently, microfluidic chip based analytical methods have high interest as a high throughput analytical platform to determine various chemical or biological samples in water based solutions [4-9]. Especially, microfluidic chip based analytical methods provide rapid determination using very small amount of sample and reagent solutions such as nano-liter level [4-9].

In this study, metal ions as a catalyst were attached to the multiwalled carbon nanotubes (MWCNTs) and a metal-MWCNTs immobilized microfluidic chip was fabricated for determination of AA. Then the AA was determined using the microfluidic chip on the optimized conditions.

\section{Experimental}

Oxidized MWCNTs were prepared using $60 \%$ nitric acid for $12 \mathrm{hrs}$ at $120{ }^{\circ} \mathrm{C}$. Then various metal ions such as $\mathrm{Co}^{2+}, \mathrm{Cu}^{2+}, \mathrm{Fe}^{3+}$ were attached on the oxidized MWCNTs respectively. The prepared metal-MWCNTs were immobilized on the microchamber of a polydimethylsiloxane (PDMS) microfluidic chip (Fig. 1a and 1b). The PDMS microfluidic chip was fabricated by soft-lithographic procedure using a microfluidic chip mold that prepared by a photolithographic procedure. The microfluidic chip have three inlets to introduce $A A$, luminol, and hydrogel peroxide solutions and $a$ microchamber having the metal-MWCNTs. The experimental conditions including concentration of luminol, hydrogen peroxide, and metal ions, $\mathrm{pH}$, flow rate of each solution were investigated and optimized. Then the $\mathrm{CL}$ intensity was measured at the $425 \mathrm{~nm}$. A spectrofluorimeter (Model F-4500, Hitachi, Japan) equipped with a photo multiplier tube was used to detect $\mathrm{CL}$ intensities from the reaction at the microchamber. While the $\mathrm{CL}$ intensity was obtaining, the light source of the 
spectrofluorimeter was turned off. A pH meter (Model Orion, 520A, USA) was used. On the optimized conditions, the determination of $A A$ was performed using the metal-MWCNTs immobilized microfluidic chip.

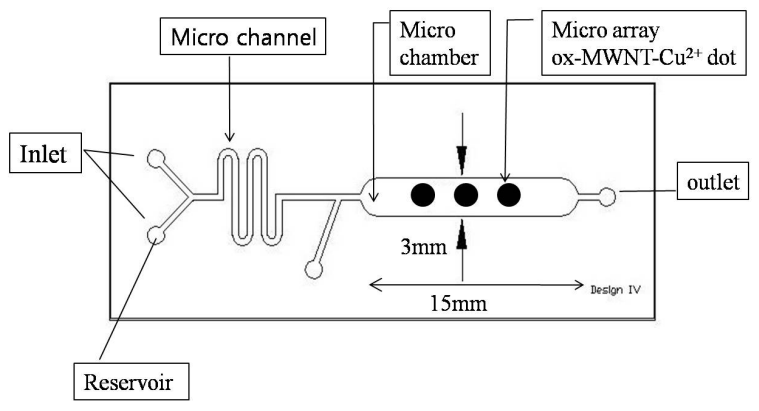

Immobilized ox-MWNT-Cu ${ }^{2+}$ dots

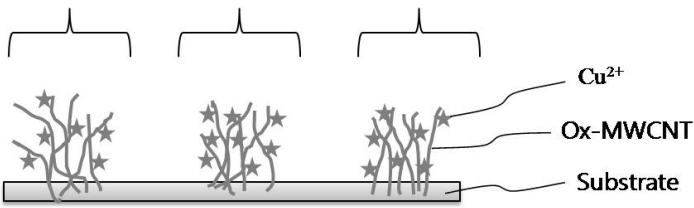

Fig. 1. Schematic diagram of a metal ion attached MWCNT immobilized microfluidic chip.

\section{Results and discussion}

Metal ions such as $\mathrm{Co}^{2+}, \mathrm{Cu}^{2+}, \mathrm{Fe}^{3+}$ have catalytic activity at luminol-hydrogen peroxide $\mathrm{CL}$ reaction that enhance $\mathrm{CL}$ intensity. Furthermore the immobilized metal-MWCNTs in the microfluidic chip was highly enhanced the $\mathrm{CL}$ intensity of the luminol-hydrogen peroxide system as shown in Fig. 2. Thus, the metalMWCNTs immobilized microfluidic chip was fabricated to determine an antioxidant such as AA. By adding an antioxidant, the $C L$ intensity of luminol system could be reduced. Therefore, a highly enhanced $\mathrm{CL}$ signal is necessary to determine a very low concentrated antioxidant sample. Among the metal ions, $\mathrm{Co}^{2+}, \mathrm{Cu}^{2+}, \mathrm{Fe}^{3+}$, attached MWCNTs, $\mathrm{Cu}^{2+}-\mathrm{MWCNTs}$ produced highest $\mathrm{CL}$ intensity. Thus, the $\mathrm{Cu}^{2+}$-MWCNTs immobilized microfluidic chip was used to determine $A A$ in a sample solution. By adding AA into the microfluidic channel of the chip, the $\mathrm{CL}$ intensity was reduced according to the concentration of $\mathrm{AA}$. Based on the results, the $A A$ was determined using the microfluidic chip as shown in Fig. 3. The linear range of the calibration curve for $A A$ was obtained from $1.6 \times 10^{-8} \mathrm{M}$ to $6.4 \times 10^{-7} \mathrm{M}$, the correction coefficient was 0.98913 and the detection limit was $1.11 \times 10^{-9} \mathrm{M}$.

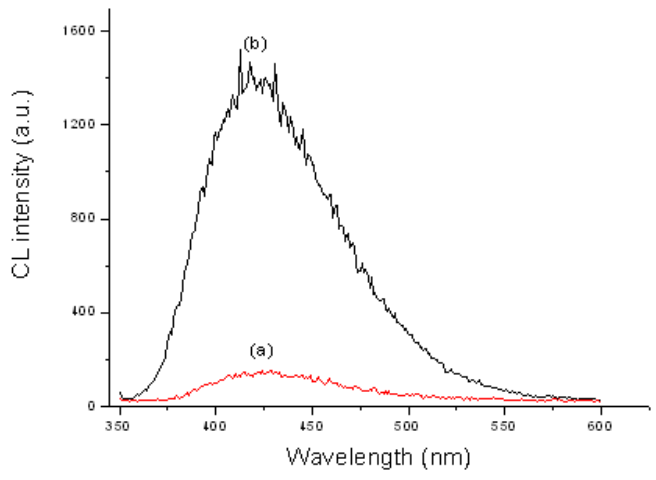

Fig. 2. $\mathrm{CL}$ spectra of : (a) [luminol], $1.2 \times 10^{-2} \mathrm{M}$, $\left[\mathrm{H}_{2} \mathrm{O}_{2}\right.$ ], $0.1 \mathrm{M} ; \mathrm{pH}, 12.8$ (b) [luminol], $1.2 \times 10^{-2} \mathrm{M}$; $\left[\mathrm{H}_{2} \mathrm{O}_{2}\right], 0.1 \mathrm{M}, \mathrm{Cu}^{2+}-$ MWCNTs dot, $10.5 \mu \mathrm{g}$.

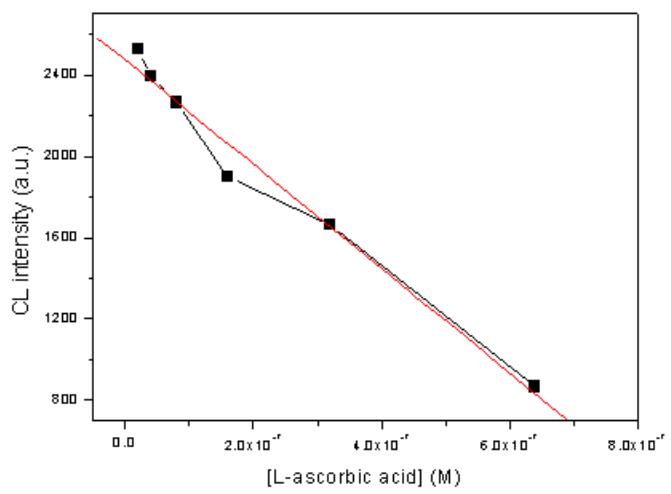

Fig. 3. Calibration curve for $A A$ obtained by chemiluminescence method using the microfluidic chip : [luminol], $1.2 \times 10^{-2} \mathrm{M} ; \mathrm{pH}, 12.8 ;\left[\mathrm{H}_{2} \mathrm{O}_{2}\right.$ ], $0.1 \mathrm{M}$; $\mathrm{Cu}^{2+}-$ MWCNTs $28 \mu \mathrm{g}$.

\section{Conclusion}

A microfluidic chip based high throughput determination of L-ascorbic acid has been presented. The immobilized metal-MWCNTs on the microchamber of the microfluidic chip highly enhanced $C L$ intensity of luminol. Thus, very low concentrated AA could be determined by using the enhanced $C L$ signal of the metalMWCNTs immobilized microfluidic chip. The metal-MWCNTs immobilized microfluidic chip could be applicable to determination of an antioxidant in a water based solution.

\section{Acknowledgements}

This work was supported by the Korea Research Council of Fundamental Science and 
Technology (KRCF) through Basic Research Project managed by the Korea Research Institute of Standards and Science (KRISS). This work was supported by the Priority Research Centers Program through the National Research Foundation of Korea (NRF) funded by the Ministry of Education, Science and Technology (2009-0093819)

\section{References}

[1] D. B. McCormick, in N. W. Tietz (Ed.), Textbook of Clinical Chemistry, Sanders, Philadelphia, PA, 959-962 (1986).

[2] L. A. Pachla, D. L. Reynolds and P. T. Kissinger, J. Assoc.Off. Anal. Chem., 68, 1 (1985); doi.org/10.1016/S0378-4347(96)00248-4

[3] P. W. Washko, R. W. Welch, K. R. Dhariwal, Y. Wang and M. Levine, Anal. Biochem., 204, 1 (1992); PII S002 1-9673(97)00465-2

[4] Y. Lv, Z. Zhang, F. Chen, Talanta, 59, 571-576 (2003).

[5] Z. X. Gao, H. F. Li, J. Liu, J. M. Lin, Analytical Chimica Acta, 622, 143-149 (2008).

[6] K. A. Heyriesa, M. G. Loughranb, D. Hoffmann, A. Homsy, L. J. Bluma, C. A. Marquettea, Biosensors \& Bioelectronics, 23, 1812-1818 (2008).

[7] V. Srinivasan, V. K. Pamula, R. B. Fair, Analytical Chimica Acta, 507, 145-150 (2004).

[8] X. B. Yin, Y. Du, X. Yang, E. Wang, Journal of Chromatography A, 1091, 158-162 (2005).

[9] D. He, Z. Zhang, Y. Huang, Y. Hu, Food Chemistry, 101, 667-672 (2007). 